\title{
THE LUTHERAN-SECRETOR LINKAGE IN MAN : SUPPORT FOR MOHR'S FINDINGS
}

\author{
RUTH SANGER and R. R. RACE \\ Medical Research Council Blood Group Research Unit, \\ The Lister Institute, London
}

Received 5.iii. $5^{8}$

THE first autosomal linkage to be recognised in Man was that between the Lutheran genes and genes responsible for the Lewis phenotype of red cells (Mohr, I95 I $a$, I95 I $b$, I954, I956). Owing presumably to the rarity of informative matings no confirmation of the linkage has appeared from other laboratories. When we last analysed our own family data for linkage (Race and Sanger, I954) we had practically nothing to contribute to the Lutheran-Lewis comparison, but since that time two very informative families have been encountered. Our collected results now bring strong support for the linkage which, in the light of our present understanding of the Lewis phenotypes, now appears to be between the Lutheran and secretor genes-a possibility that had been appreciated by Mohr.

\section{GENES}

(i) The Lutheran genes

Nothing complicated is yet known about the Lutheran genes : $L u^{a}$ in single dose or in double dose causes the presence on the red cells of the antigen $\mathrm{Lu}^{\mathrm{a}}$. The antigen $\mathrm{Lu}^{\mathrm{b}}$, and therefore the gene $L u^{b}$, was shown to have a positive existence when, in 1956, Cutbush and Chanarin discovered the antibody anti-Lu ${ }^{\mathrm{b}}$.

\section{(ii) The secretor genes}

The presence or absence of water-soluble $\mathrm{ABH}$ substance in the saliva still appears relatively straightforward. The secretor genes, Se se, so modify the action of the $A B O$ genes that when the genotype is se se practically no $\mathrm{ABH}$ substance is to be found in the saliva, whereas large quantities of the appropriate substances are present when the genotype is $\mathrm{Se} S e$ or $\mathrm{Se}$ se. (The antigen $\mathrm{H}$ is not understood; it is certainly not the product of the gene $O$, but its presence or absence in the saliva of people of group $\mathrm{O}$ serves to classify them as secretors or non-secretors.) The words secretor and non-secretor refer to the presence or absence of the $\mathrm{ABH}$ substances alone; they should not be used for the presence or absence of other substances-such as Le ${ }^{a}$ which has a quite different genetic background. 
(iii) The Lewis genes

The inheritance of the Lewis antigens is, on the other hand, most complex. Our present views are based mainly on the work of Grubb (1948, I950, I95 I), Ceppellini (1955a, I 955b), Ceppellini and Siniscalco (1955), Sneath and Sneath (r955), and Mäkelä and Mäkelä (r956).

The antigen Le ${ }^{a}$ is thought to be primarily an antigen of the body fluids, notably of the saliva, which only secondarily becomes hitched onto the red cells (Grubb, I950; Ceppellini, I955a, I955b; Sneath

TABLE I

Scheme of the Lewis system and secretion of $A B H$

(Slightly modified, from Grubb, 195 I and Ceppellini, 1955)

\begin{tabular}{|c|c|c|c|}
\hline \multirow{3}{*}{ Genotypes } & \multicolumn{3}{|c|}{ Phenotypes } \\
\hline & \multicolumn{2}{|c|}{ Saliva } & \multirow{2}{*}{ Red cells } \\
\hline & $\mathrm{ABH}$ & $\mathrm{Le}^{\mathrm{a}}$ & \\
\hline $\begin{array}{l}\text { Se Se LL } \\
\text { Se Se Ll } \\
\text { Se se LL } \\
\text { Se se Ll }\end{array}$ & + & + & $\operatorname{Le}(a-b+)$ \\
\hline $\begin{array}{l}\text { se se } L L \\
\text { se se } L l\end{array}$ & - & + & $\operatorname{Le}(a+b-)$ \\
\hline $\begin{array}{l}\text { Se Sell } \\
\text { Se sell }\end{array}$ & + & - & $\operatorname{Le}(a-b-)$ \\
\hline se sell & - & - & $\operatorname{Le}(a-b-)$ \\
\hline
\end{tabular}

and Sneath, 1955). The gene which produces the antigen has been called $L$; it is dominant in its effect and is not linked to the secretor or $A B O$ genes (Ceppellini, I955a ; Ceppellini and Siniscalco, 1955).

The Lewis phenotype of the red cells is thought to be the result of interaction between the $L l$ genes and the secretor genes as shown in table I. It seems that there is a limited amount of substrate that can be made into water-soluble $\mathrm{ABH}$ substance or $\mathrm{Le}^{\mathrm{a}}$ substance. The demands of the $A, B$ and $O$ and secretor genes are satisfied first and, consequently, in secretors but little substrate is left for the $L$ gene to mould into Le ${ }^{a}$ substance : some Le ${ }^{a}$ substance does reach the saliva but not enough reaches the plasma to hook onto the red cells and make them give the $\operatorname{Le}(\mathrm{a}+)$ reaction. When the $A B O$ and secretor genes require none of this substrate (that is in non-secretors) all of it is available for moulding into Le ${ }^{\text {a }}$ substance by the gene $L$; then the saliva is saturated with Le ${ }^{a}$ substance and enough reaches the plasma to hook on to the red cells and make them give the $\operatorname{Le}(\mathrm{a}+)$ reaction. 
There does not seem to be an $L e^{\delta}$ gene, and the red cell antigen $L^{b}$ appears to be a product of the interaction of the $A B O$ genes, the secretor gene $S e$ and the gene $L$. A further complication is that the reactions of anti-Le ${ }^{b}$ sera are influenced by the $A B O$ group of the red cells : using most anti-Le ${ }^{b}$ sera too many $A_{1}$ and $A_{1} B$ red cells are classified as Le $(\mathrm{b}-)$.

Nevertheless, as a result of the interaction of the Lewis and secretor genes the red cell phenotype $\mathrm{Le}(\mathrm{a}+)$ behaves like a recessive character for it reflects the presence of two se genes and the following relations hold :

$\mathrm{Le}(\mathrm{a}+\mathrm{b}-)$ red cells belong to non-secretors,

Le $(a-b+)$ red cells belong to secretors,

Le $(a-b-)$ red cells usually, but not always, belong to secretors.

Consequently when no saliva has been available we can confidently assume that all people whose red cells give the reaction $\operatorname{Le}(\mathrm{a}+)$ are non-secretors and that those whose red cells give the reaction $\mathrm{Le}(\mathrm{a}-\mathrm{b}+)$ are secretors. We would usually be right in assuming that the 6 per cent. or so of white people whose red cells give the reaction $\operatorname{Le}(\mathrm{a}-\mathrm{b}-)$ are secretors.

\section{LINKAGE DATA}

Table 2 contains all the families of two or more children whose red cells we have tested with ant-Lu ${ }^{a}$ and anti-Le a and in which a parent is $\mathrm{Lu}(\mathrm{a}+)$. When samples of saliva were available they have been classified as secretor or non-secretor ; most of them have been further tested for Le ${ }^{a}$ substance.

In the analysis the $u$ statistics of Fisher (I935) have been used as elaborated by Finney (1940). Only "certain" families have been scored and they have been treated as Finney's Mating Types I 3 and I4 (in his table I). Finney's symbols have been translated thus: $\mathrm{W}=\mathrm{Lu}(\mathrm{a}+)$ and $\mathrm{w}=\mathrm{Lu}(\mathrm{a}-) ; \mathrm{T}=$ secretor, Le ${ }^{\mathrm{a}}$ present in saliva $(\mathrm{L})$, or $\mathrm{Le}(\mathrm{a}-)$ phenotype of red cells, $\mathrm{t}=$ non-secretor, $\mathrm{Le}^{\mathrm{a}}$ absent from saliva (ll), or Le(a + ) phenotype of red cells, according to the characters being analysed. In one comparison, that between secretor and L, Mating Type I 5 has been used.

The results of the various comparisons are given in table 3. The last column but one shows the significance of the results: Finney states that if $\Sigma(\lambda)$ exceeds $2 \cdot 33 \sqrt{\Sigma(\kappa)}$ linkage is established at the I in roo level of probability. Linkage having been established, the last column shows the estimate of the recombination fraction which, again according to Finney, is $\frac{1}{2}\left[\mathrm{I}-\sqrt{\frac{\Sigma(\lambda)}{\Sigma(\kappa)}}\right]$.

In table 4 the latest results of Mohr (1954, I956) and of Ceppellini and Siniscalco (I955) are added to our own. 
TABLE 2-The families

\begin{tabular}{|c|c|c|c|c|c|c|c|c|c|}
\hline \multirow{2}{*}{ No. } & \multirow{2}{*}{ Father } & \multirow{2}{*}{ Mother } & \multicolumn{7}{|c|}{ Children } \\
\hline & & & $x$ & 2 & 3 & 4 & 5 & 6 & 7 \\
\hline I & $\begin{array}{c}\mathrm{Lu}(\mathrm{a}-) \\
\text { non-sec. } \\
\mathrm{Le}(\mathrm{a}+\mathrm{b}-\mathrm{b}) \\
\mathrm{L} \\
\mathrm{A}_{1}\end{array}$ & $\begin{array}{c}\mathrm{Lu}(\mathrm{a}+) \\
\text { sec. } \\
\mathrm{Le}(\mathrm{a}-\mathrm{b}+) \\
\mathrm{L} \\
\mathrm{O}\end{array}$ & $\begin{array}{c}\mathrm{Lu}(\mathrm{a}-) \\
\text { sec. } \\
\mathrm{Le}(\mathrm{a}-\mathrm{b}+) \\
\mathrm{L} \\
\mathrm{A}_{1} \\
\end{array}$ & $\begin{array}{c}\operatorname{Lu}(a-) \\
\text { sec. } \\
\operatorname{Le}(a-b+) \\
L \\
A_{1} \\
\end{array}$ & $\begin{array}{c}\mathrm{Lu}(\mathrm{a}-) \\
\text { sec. } \\
\mathrm{Le}(\mathrm{a}-\mathrm{b}+) \\
\mathrm{L} \\
\mathrm{O} \\
\end{array}$ & $\begin{array}{c}\mathrm{Lu}(\mathrm{a}+) \\
\text { non-sec. } \\
\mathrm{Le}(\mathrm{a}+\mathrm{b}-) \\
\mathrm{L} \\
\mathrm{O} \\
\end{array}$ & $\begin{array}{c}\mathrm{Lu}(\mathrm{a}-) \\
\sec \\
\mathrm{Le}(\mathrm{a}-\mathrm{b}+) \\
\mathrm{L} \\
\mathrm{A}_{1} \\
\end{array}$ & \begin{tabular}{|c|}
$\operatorname{Lu}(a-)$ \\
$\sec$ \\
$\mathrm{Le}(a-b+)$ \\
$\mathrm{L}$ \\
$\mathrm{O}$
\end{tabular} & $\begin{array}{l}\operatorname{Lu}(a+) \\
\text { sec. } \\
\operatorname{Le}(a-b+) \\
L \\
A_{1}\end{array}$ \\
\hline 2 & $\begin{array}{c}\mathrm{Lu}(\mathrm{a}-\mathrm{b}+) \\
\text { non-sec. } \\
\mathrm{Le}(\mathrm{a}+\mathrm{b}-) \\
\mathrm{L} \\
\mathrm{O}\end{array}$ & \begin{tabular}{|c|}
$\mathrm{Lu}(\mathrm{a}+)$ \\
sec. \\
$\mathrm{Le}(\mathrm{a}-\mathrm{b}+)$ \\
$\mathrm{L}$ \\
$\mathrm{O}$
\end{tabular} & $\begin{array}{c}\text { Lu }(a+b+) \\
\text { non-sec. } \\
\text { Le }(a+b-) \\
\text { L } \\
O\end{array}$ & \begin{tabular}{|c|} 
Lu(a+b+) \\
non-sec. \\
Le $(a+b-)$ \\
L \\
O \\
\end{tabular} & \begin{tabular}{|c|}
$\mathrm{Lu}(\mathrm{a}+\mathrm{b}+)$ \\
non-sec. \\
$\mathrm{Le}(\mathrm{a}+\mathrm{b}-)$ \\
$\mathrm{L}$ \\
$\mathrm{O}$ \\
\end{tabular} & \begin{tabular}{|c|}
$\mathrm{Lu}(a-b+)$ \\
sec. \\
$\mathrm{Le}(a-b+)$ \\
$\mathrm{L}$ \\
$\mathrm{O}$
\end{tabular} & \begin{tabular}{|c|}
$\mathrm{Lu}(\mathrm{a}+\mathrm{b}+)$ \\
non.sec. \\
$\mathrm{Le}(\mathrm{a}+\mathrm{b}-)$ \\
$\mathrm{L}$ \\
$\mathrm{O}$
\end{tabular} & & \\
\hline 3 & \begin{tabular}{|c|}
$\mathrm{Lu}(\mathrm{a}+)$ \\
sec. \\
$\mathrm{Le}(\mathrm{a}-\mathrm{b}-)$ \\
!l \\
$\mathrm{O}$ \\
\end{tabular} & \begin{tabular}{|c|}
$\mathrm{Lu}(\mathrm{a}-)$ \\
non-sec. \\
$\mathrm{Le}(\mathrm{a}+\mathrm{b}-\mathbf{b})$ \\
$\mathrm{L}$ \\
$\mathrm{A}_{1}$
\end{tabular} & $\begin{array}{c}\operatorname{Lu}(a+) \\
\operatorname{Le}(a-b+) \\
0 \\
\end{array}$ & $\begin{array}{c}\text { Lu(a-) } \\
\text { non-sec. } \\
\text { Le(a }(a-b-) \\
\text { L } \\
\text { O }\end{array}$ & $\begin{array}{c}\operatorname{Lu}(a-) \\
\text { sec. } \\
\text { Le(a-b+) } \\
L \\
O \\
\end{array}$ & & & & \\
\hline 4 & $\begin{array}{c}\mathrm{Lu}(\mathrm{a}+) \\
\mathrm{Le}(\mathrm{a}-\mathrm{b}+) \\
\mathrm{O}\end{array}$ & $\begin{array}{c}\mathrm{Lu}(\mathrm{a}-\mathrm{-} \\
\mathrm{Le}(\mathrm{a}+\mathrm{b}-) \\
\mathrm{A}_{1}\end{array}$ & $\begin{array}{c}\operatorname{Lu}(a+) \\
\operatorname{Le}(a-b+) \\
A_{1} \\
\end{array}$ & $\begin{array}{c}\mathrm{Lu}(\mathrm{a}-) \\
\mathrm{Le}(\mathrm{a}+\mathrm{b}-) \\
\mathrm{O}\end{array}$ & & & & & \\
\hline 5 & $\begin{array}{c}\operatorname{Lu}(a+) \\
\operatorname{Le}(a-b+) \\
O\end{array}$ & $\begin{array}{c}\operatorname{Lu}(a-) \\
\operatorname{Le}(a+b-) \\
A_{1}\end{array}$ & $\begin{array}{c}\mathrm{Lu}(\mathrm{a}-) \\
\mathrm{Le}(\mathrm{a}+\mathrm{b}-) \\
\mathrm{O}\end{array}$ & $\begin{array}{c}\mathrm{Lu}(\mathrm{a}-) \\
\mathrm{Le}(\mathrm{a}+\mathrm{b}-) \\
\mathrm{A}_{1}\end{array}$ & $\begin{array}{c}\operatorname{Lu}(a-) \\
\operatorname{Le}(a+b-) \\
A_{1}\end{array}$ & \multicolumn{2}{|c|}{ identical twins } & & \\
\hline 6 & $\begin{array}{c}\mathrm{Lu}(\mathrm{a}-) \\
\text { non-sec. } \\
\text { Le }(\mathrm{a}+\mathrm{b}-) \\
\mathrm{L} \\
\mathrm{B} \\
\end{array}$ & \begin{tabular}{|c|}
$\operatorname{Lu}(a+)$ \\
sec. \\
Le $(a-b+)$ \\
$L$ \\
$A_{1}$ \\
\end{tabular} & $\begin{array}{c}\mathrm{Lu}(\mathrm{a}+) \\
\text { sec. } \\
\mathrm{Le}(\mathrm{a}-) \\
\mathrm{L} \\
\mathrm{A}_{1} \mathrm{~B} \\
\end{array}$ & $\begin{array}{c}\text { Lu(a-) } \\
\text { sec. } \\
\text { Le(a-) } \\
\text { L } \\
A_{1} B\end{array}$ & $\begin{array}{c}\mathrm{Lu}(\mathrm{a}+) \\
\text { sec. } \\
\mathrm{Le}(\mathrm{a}-\mathrm{b}+) \\
\mathrm{L} \\
\mathrm{O} \\
\end{array}$ & & & & \\
\hline 7 & $\begin{array}{c}\operatorname{Lu}(a+) \\
\operatorname{Le}(a-b+) \\
A_{1}\end{array}$ & $\begin{array}{c}\operatorname{Lu}(a-) \\
\operatorname{Le}(a-b+) \\
O\end{array}$ & $\begin{array}{c}\operatorname{Lu}(a-) \\
\operatorname{Le}(a-b+) \\
A_{1}\end{array}$ & $\begin{array}{c}\operatorname{Lu}(a+) \\
\operatorname{Le}(a+b-) \\
O\end{array}$ & & & & & \\
\hline 8 & $\begin{array}{c}\mathrm{Lu}(\mathrm{a}-) \\
\mathrm{Le}(\mathrm{a}-\mathrm{b}+) \\
\mathrm{A}_{1}\end{array}$ & $\begin{array}{c}\operatorname{Lu}(a+) \\
\operatorname{Le}(a-b+) \\
A_{1}\end{array}$ & $\begin{array}{c}\operatorname{Lu}(a-) \\
\operatorname{Le}(a+b-) \\
A_{1}\end{array}$ & $\begin{array}{c}\operatorname{Lu}(a-) \\
\operatorname{Le}(a-b+) \\
A_{1}\end{array}$ & & & & & \\
\hline 9 & $\begin{array}{c}\mathrm{Lu}(\mathrm{a}-) \\
\mathrm{Le}(\mathrm{a}-) \\
\mathrm{O}\end{array}$ & $\begin{array}{l}\operatorname{Lu}(a+)^{*} \\
\operatorname{Le}(a-) \\
O\end{array}$ & $\begin{array}{c}\mathrm{Lu}(\mathrm{a}+) \\
\mathrm{Le}(\mathrm{a}+) \\
\mathrm{O}\end{array}$ & $\begin{array}{c}\operatorname{Lu}(a+) \\
\operatorname{Le}(a-) \\
O\end{array}$ & & & & & \\
\hline xo & $\begin{array}{r}\mathrm{Lu}(\mathrm{a}+) \\
\mathrm{Le}(\mathrm{a}-) \\
\mathrm{A}_{\mathbf{2}} \mathrm{B}\end{array}$ & $\begin{array}{c}\operatorname{Lu}(a-) \\
\operatorname{Le}(a-) \\
A_{1}\end{array}$ & $\begin{array}{r}\operatorname{Lu}(a-) \\
\operatorname{Le}(a-) \\
A_{1} B\end{array}$ & $\begin{array}{c}\text { Lu(a+) } \\
\text { Le(a+) } \\
B\end{array}$ & & & & & \\
\hline$x x$ & $\begin{array}{c}\mathrm{Lu}(\mathrm{a}-) \\
\mathrm{sec} . \\
\mathrm{Le}(\mathrm{a}-) \\
\mathrm{A}_{1}\end{array}$ & $\begin{array}{c}\mathrm{Lu}(\mathrm{a}+) \\
\text { sec. } \\
\mathrm{Le}(\mathrm{a}-) \\
\mathrm{A}_{\mathrm{I}}\end{array}$ & $\begin{array}{c}\mathrm{Lu}(\mathrm{a}-) \\
\text { sec. } \\
\mathrm{Le}(\mathrm{a}-) \\
\mathrm{A}_{1}\end{array}$ & $\begin{array}{c}\mathrm{Lu}(\mathrm{a}+) \\
\text { non-sec. } \\
\mathrm{Le}(\mathrm{a}+) \\
\mathrm{A}_{1}\end{array}$ & $\begin{array}{c}\mathrm{Lu}(\mathrm{a}+) \\
\mathrm{Le}(\mathrm{a}-) \\
\mathrm{A}_{1}\end{array}$ & $\begin{array}{c}\mathrm{Lu}(\mathrm{a}+) \\
\text { sec. } \\
\mathrm{Le}(\mathrm{a}-) \\
\mathrm{A}_{1}\end{array}$ & & & \\
\hline I2 & $\begin{array}{c}\mathrm{Lu}(\mathrm{a}-) \\
\operatorname{Le}(\mathrm{a}-) \\
A_{1}\end{array}$ & $\begin{array}{c}\operatorname{Lu}(a+) \\
\operatorname{Le}(a-) \\
B\end{array}$ & $\begin{array}{r}\mathrm{Lu}(\mathrm{a}+) \\
\mathrm{Le}(\mathrm{a}-\mathrm{f}) \\
\mathrm{A}_{1} \mathrm{~B}\end{array}$ & $\begin{array}{c}\operatorname{Lu}(a-) \\
\operatorname{Le}(a+) \\
A_{1}\end{array}$ & $\begin{array}{r}\mathrm{Lu}(\mathrm{a}+) \\
\mathrm{Le}(\mathrm{a}-) \\
\mathrm{A}_{1} \mathrm{~B}\end{array}$ & & & & \\
\hline$x_{3}$ & \begin{tabular}{|c}
$\operatorname{Lu}(a+)$ \\
sec. \\
$\operatorname{Le}(a-b+)$ \\
$L$ \\
$A_{1}$ \\
\end{tabular} & \begin{tabular}{|c|}
$\mathrm{Lu}(\mathrm{a}-)$ \\
sec. \\
$\mathrm{Le}(\mathrm{a}-\mathrm{b}+)$ \\
$\mathrm{L}$ \\
$\mathrm{A}_{2}$
\end{tabular} & $\begin{array}{c}\mathrm{Lu}(\mathrm{a}-\mathrm{-} \\
\text { sec. } \\
\mathrm{Le}(\mathrm{a}-\mathrm{b}+) \\
\mathrm{L} \\
\mathrm{A}_{2}\end{array}$ & \begin{tabular}{|c|}
$\operatorname{Lu}(a+)$ \\
sec. \\
Le(a-b+) \\
$\mathrm{L}$ \\
$\mathrm{A}_{1}$ \\
\end{tabular} & $\begin{array}{c}\mathrm{Lu}(\mathrm{a}-) \\
\text { non-sec. } \\
\mathrm{Le}(\mathrm{a}-\mathrm{b}-) \\
\mathrm{ll} \\
\mathrm{A}_{2} \\
\end{array}$ & \begin{tabular}{|c|}
$\mathrm{Lu}(\mathrm{a}-)$ \\
sec. \\
$\mathrm{Le}(\mathrm{a}-\mathrm{b}+)$ \\
$\mathrm{L}$ \\
$\mathrm{A}_{1}$ \\
\end{tabular} & $\begin{array}{c}\operatorname{Lu}(a+) \\
\text { sec. } \\
\operatorname{Le}(a-b-) \\
11 \\
A_{2} \\
\end{array}$ & \begin{tabular}{|c|}
$\mathrm{Lu}(\mathrm{a}-)$ \\
sec. \\
$\mathrm{Le}(\mathrm{a}-\mathrm{b}+)$ \\
$\mathrm{L}$ \\
$\mathrm{A}_{1}$ \\
\end{tabular} & \\
\hline$\sqrt{4}$ & $\begin{array}{c}\mathrm{Lu}(\mathrm{a}+\mathrm{f} \\
\text { sec. } \\
\mathrm{Le}(\mathrm{a}-\mathrm{b}+) \\
\mathrm{L} \\
\mathrm{O}\end{array}$ & $\begin{array}{c}\mathrm{Lu}(\mathrm{a}-) \\
\text { sec. } \\
\mathrm{Le}(\mathrm{a}-\mathrm{b}+) \\
\mathrm{L} \\
\mathrm{O}\end{array}$ & \begin{tabular}{|c|} 
Lu(a-) \\
sec. \\
Le $(a-b+)$ \\
$L$ \\
$O$
\end{tabular} & \begin{tabular}{|c|} 
Lu(a+d) \\
sec. \\
Le $(a-b+)$ \\
L \\
$O$
\end{tabular} & & & & & \\
\hline I5 & \begin{tabular}{|c|} 
Lu(a-) \\
sec. \\
Le $(a-b+)$ \\
L \\
$O$
\end{tabular} & \begin{tabular}{|c|} 
Lu(a +$)$ \\
sec. \\
Le $(a-b+)$ \\
L \\
B \\
\end{tabular} & $\begin{array}{c}\operatorname{Lu}(a-) \\
\text { sec. } \\
\text { Le }(a-b+) \\
\text { L } \\
\text { B } \\
\end{array}$ & \begin{tabular}{|c|}
$\mathrm{Lu}(\mathrm{a}-)$ \\
sec. \\
$\mathrm{Le}(\mathrm{a}-\mathrm{b}+)$ \\
$\mathrm{L}$ \\
$\mathrm{B}$ \\
\end{tabular} & & & & & \\
\hline 16 & $\begin{array}{c}\text { Lu(a-) } \\
\operatorname{Le}(a-b+) \\
B\end{array}$ & $\begin{array}{c}\operatorname{Lu}(a+) \\
\operatorname{Le}(a-b+) \\
B\end{array}$ & $\begin{array}{c}\operatorname{Lu}(a-) \\
\operatorname{Le}(a-b+) \\
B\end{array}$ & $\begin{array}{c}\operatorname{Lu}(a+) \\
\operatorname{Le}(a-b+) \\
O\end{array}$ & & & & & \\
\hline 17 & $\begin{array}{c}\operatorname{Lu}(a-) \\
\operatorname{Le}(a-b+) \\
A_{1}\end{array}$ & $\begin{array}{c}\mathrm{Lu}(\mathrm{a}+) \\
\mathrm{Le}(a-b+) \\
A_{1}\end{array}$ & $\begin{array}{c}\mathrm{Lu}(a-) \\
\mathrm{Le}(a-b+) \\
A_{1}\end{array}$ & $\begin{array}{c}\operatorname{Lu}(a+) \\
\operatorname{Le}(a-b+) \\
A_{1}\end{array}$ & & & & & \\
\hline 18 & $\begin{array}{c}\text { Lu }(a-) \\
\operatorname{Le}(a-b+) \\
O\end{array}$ & $\begin{array}{c}\operatorname{Lu}(a+) \\
\operatorname{Le}(a-b+) \\
A_{1}\end{array}$ & $\begin{array}{c}\operatorname{Lu}(a+) \\
\operatorname{Le}(a-b+) \\
A_{1}\end{array}$ & $\begin{array}{c}\mathrm{Lu}(\mathrm{a}-) \\
\operatorname{Le}(a-b+) \\
O\end{array}$ & & & & & \\
\hline 19 & \begin{tabular}{|c|}
$\operatorname{Lu}(a-)$ \\
sec. \\
$\operatorname{Le}(a-b+)$ \\
$L$ \\
$A_{1}$ \\
\end{tabular} & \begin{tabular}{|c|} 
Lu(a +$)$ \\
sec. \\
Le(a-b+) \\
L \\
O \\
\end{tabular} & $\begin{array}{c}\operatorname{Lu}(a+) \\
\operatorname{Le}(a-b-) \\
0 \\
\end{array}$ & $\begin{array}{c}\mathrm{Lu}(\mathrm{a}-) \\
\text { sec. } \\
\mathrm{Le}(\mathrm{a}-\mathrm{b}+) \\
\mathrm{L} \\
\mathrm{O} \\
\end{array}$ & $\begin{array}{c}\mathrm{Lu}(\mathrm{a}-) \\
\text { sec. } \\
\mathrm{Le}(\mathrm{a}-\mathrm{b}-) \\
11 \\
\mathrm{~A}_{1} \\
\end{array}$ & & & & \\
\hline 20 & $\begin{array}{c}\operatorname{Lu}(a-) \\
\text { sec. } \\
\operatorname{Le}(a-b+) \\
\text { L } \\
O\end{array}$ & \begin{tabular}{|} 
Lu(a+) \\
sec. \\
Le(a-b+) \\
$L$ \\
$A_{1}$
\end{tabular} & \begin{tabular}{|c|} 
Lu(a-) \\
sec. \\
Le(a-b+) \\
L \\
$O$
\end{tabular} & $\begin{array}{c}\text { Lu(a-) } \\
\text { sec. } \\
\text { Le(a-b+) } \\
L \\
A_{1}\end{array}$ & $\begin{array}{c}\operatorname{Lu}(a+) \\
\sec . \\
\operatorname{Le}(a-b+) \\
L \\
O\end{array}$ & $\begin{array}{c}\mathrm{Lu}(\mathrm{a}+\mathbf{)} \\
\mathrm{Le}(\mathrm{a}-\mathrm{b}+) \\
\mathrm{O}\end{array}$ & & & \\
\hline
\end{tabular}


TABLE 2-continued

\begin{tabular}{|c|c|c|c|c|c|c|c|c|c|}
\hline \multirow{2}{*}{ No. } & \multirow{2}{*}{ Father } & \multirow{2}{*}{ Mother } & \multicolumn{7}{|c|}{ Children } \\
\hline & & & I & 2 & 3 & 4 & 5 & 6 & 7 \\
\hline $2 I$ & $\begin{array}{c}\operatorname{Lu}(a-) \\
\operatorname{Le}(a-b+) \\
A_{2}\end{array}$ & $\begin{array}{l}\operatorname{Lu}(a+) \\
\operatorname{Le}(a-b+) \\
A_{2} B\end{array}$ & $\begin{array}{c}\operatorname{Lu}(a+) \\
\operatorname{Le}(a-b+) \\
A_{1}\end{array}$ & $\begin{array}{c}\operatorname{Lu}(a-) \\
\operatorname{Le}(a-b+) \\
B\end{array}$ & $\begin{array}{l}\operatorname{Lu}(a-) \\
\operatorname{Le}(a-b+) \\
\quad A_{1} B\end{array}$ & & & & \\
\hline 22 & $\begin{array}{c}\operatorname{Lu}(a+) \\
\operatorname{Le}(a-) \\
O\end{array}$ & $\begin{array}{c}\operatorname{Lu}(a-) \\
\operatorname{Le}(a-) \\
O\end{array}$ & $\begin{array}{c}\operatorname{Lu}(a-) \\
\operatorname{Le}(a-) \\
O\end{array}$ & $\begin{array}{c}\text { Lu(a+) } \\
\text { Le }(a-) \\
O\end{array}$ & & & & & \\
\hline 23 & $\begin{array}{c}\mathrm{Lu}(\mathrm{a}-) \\
\mathrm{Le}(\mathrm{a}-) \\
\mathrm{O}\end{array}$ & $\begin{array}{c}\operatorname{Lu}(a+) \\
\operatorname{Le}(a-) \\
A_{1}\end{array}$ & $\begin{array}{c}\operatorname{Lu}(a-) \\
\operatorname{Le}(a-) \\
A_{1}\end{array}$ & $\begin{array}{c}\operatorname{Lu}(a-) \\
\operatorname{Le}(a-) \\
A_{1}\end{array}$ & & & & & \\
\hline 24 & $\begin{array}{c}\operatorname{Lu}(a-) \\
\operatorname{Le}(a-) \\
A_{1}\end{array}$ & $\begin{array}{c}\mathrm{Lu}(\mathrm{a}+) \\
\mathrm{Le}(\mathrm{a}-\mathrm{)} \\
\mathrm{O}\end{array}$ & $\begin{array}{c}\operatorname{Lu}(a+) \\
\operatorname{Le}(a-) \\
A_{2}\end{array}$ & $\begin{array}{c}\operatorname{Lu}(a+) \\
\operatorname{Le}(a-) \\
A_{3}\end{array}$ & & & & & \\
\hline 25 & $\begin{array}{c}\operatorname{Lu}(a-) \\
\text { sec. } \\
\text { Le }(a-b+) \\
\text { L } \\
B\end{array}$ & $\begin{array}{c}\operatorname{Lu}(a+) \\
\text { sec. } \\
\text { Le }(a-b+) \\
L \\
O\end{array}$ & $\begin{array}{c}\mathrm{Lu}(a+) \\
\operatorname{Le}(a-b+) \\
0 \\
\end{array}$ & $\begin{array}{c}\operatorname{Lu}(a+) \\
\text { sec. } \\
\text { Le }(a-b+) \\
\text { L } \\
B\end{array}$ & & & & & \\
\hline 26 & $\begin{array}{c}\operatorname{Lu}(a+) \\
\operatorname{Le}(a-b+) \\
O\end{array}$ & $\begin{array}{c}\operatorname{Lu}(a+) \\
\operatorname{Le}(a-b+) \\
A_{1} \\
\end{array}$ & $\begin{array}{c}\operatorname{Lu}(a-) \\
\operatorname{Le}(a-b+) \\
O \\
\end{array}$ & $\begin{array}{c}\operatorname{Lu}(a+) \\
\operatorname{Le}(a-b+) \\
A_{1}\end{array}$ & & & & & \\
\hline 27 & $\begin{array}{c}\operatorname{Lu}(a+) \\
\operatorname{Le}(a-b+) \\
B\end{array}$ & $\begin{array}{c}\operatorname{Lu}(a+) \\
\text { Le }(a-b+) \\
0 \\
\end{array}$ & $\begin{array}{c}\mathrm{Lu}(a+) \\
\mathrm{Le}(a-b+) \\
0 \\
\end{array}$ & $\begin{array}{c}\mathrm{Lu}(a+) \\
\text { sec. } \\
\mathrm{Le}(\mathrm{a}-\mathrm{b}+) \\
\mathrm{L} \\
\mathrm{B} \\
\end{array}$ & & & & & \\
\hline 28 & $\begin{array}{c}\operatorname{Lu}(a+b+) \\
\operatorname{Le}(a-b-) \\
O\end{array}$ & $\begin{array}{c}\operatorname{Lu}(a+b+) \\
\operatorname{Le}(a-b+) \\
O\end{array}$ & $\begin{array}{c}\operatorname{Lu}(a+b+) \\
\operatorname{Le}(a-b+) \\
O\end{array}$ & $\begin{array}{c}\mathrm{Lu}(\mathrm{a}+) \\
\mathrm{O} \\
\end{array}$ & $\begin{array}{c}\operatorname{Lu}(a+b+) \\
\operatorname{Le}(a-b+) \\
O\end{array}$ & $\begin{array}{c}\operatorname{Lu}(a+b-) \\
\operatorname{Le}(a-b+) \\
O\end{array}$ & & & \\
\hline 29 & $\begin{array}{c}\operatorname{Lu}(a+) \\
\operatorname{Le}(a-b+) \\
O\end{array}$ & $\begin{array}{c}\operatorname{Lu}(a+) \\
\operatorname{Le}(a-) \\
A_{1}\end{array}$ & $\begin{array}{c}\operatorname{Lu}(a+) \\
\operatorname{Le}(a-b+) \\
O\end{array}$ & $\begin{array}{c}\operatorname{Lu}(a+) \\
\operatorname{Le}(a-b+) \\
O\end{array}$ & & & & & \\
\hline 30 & $\begin{array}{c}\mathrm{Lu}(a-) \\
\operatorname{Le}(a-b+) \\
\quad B\end{array}$ & $\begin{array}{c}\operatorname{Lu}(a+) \\
\operatorname{Le}(a+b-) \\
O\end{array}$ & $\begin{array}{c}\operatorname{Lu}(a+) \\
\operatorname{Le}(a-b+) \\
B\end{array}$ & $\begin{array}{c}\operatorname{Lu}(a+) \\
\operatorname{Le}(a+b-) \\
B \\
\end{array}$ & & & & & \\
\hline $3 I$ & $\begin{array}{c}\operatorname{Lu}(a-) \\
\operatorname{Le}(a-b+) \\
B\end{array}$ & $\begin{array}{c}\mathrm{Lu}(\mathrm{a}+) \\
\mathrm{Le}(\mathrm{a}+\mathrm{b}-) \\
\mathrm{B}\end{array}$ & $\begin{array}{c}\operatorname{Lu}(a+) \\
\operatorname{Le}(a+b-) \\
B\end{array}$ & $\begin{array}{c}\operatorname{Lu}(a+) \\
\operatorname{Le}(a+b-) \\
B\end{array}$ & & & & & \\
\hline 32 & $\begin{array}{c}\operatorname{Lu}(a-) \\
\operatorname{Le}(a-b+) \\
A_{1}\end{array}$ & $\begin{array}{c}\operatorname{Lu}(a+) \\
\operatorname{Le}(a+b-) \\
O\end{array}$ & $\begin{array}{c}\operatorname{Lu}(a+) \\
\operatorname{Le}(a-b+) \\
O\end{array}$ & $\begin{array}{c}\operatorname{Lu}(a+) \\
\operatorname{Le}(a-b+) \\
O\end{array}$ & & & & & \\
\hline 33 & $\begin{array}{c}\operatorname{Lu}(a-) \\
\operatorname{Le}(a-b-) \\
O\end{array}$ & $\begin{array}{c}\operatorname{Lu}(a+) \\
\operatorname{Le}(a+b-) \\
0 \\
\end{array}$ & $\begin{array}{c}\operatorname{Lu}(a+) \\
\operatorname{Le}(a-b+) \\
O \\
\end{array}$ & $\begin{array}{c}\operatorname{Lu}(a+) \\
\operatorname{Le}(a-b+) \\
O\end{array}$ & & & & & \\
\hline 34 & $\begin{array}{c}\operatorname{Lu}(a-) \\
\operatorname{Le}(a-) \\
A_{1}\end{array}$ & $\begin{array}{c}\operatorname{Lu}(a+) \\
\operatorname{Le}(a+) \\
A_{2}\end{array}$ & $\begin{array}{c}\operatorname{Lu}(a-) \\
\operatorname{Le}(a+) \\
A_{1}\end{array}$ & $\begin{array}{c}\operatorname{Lu}(a+) \\
\operatorname{Le}(a+) \\
A_{1}\end{array}$ & $\begin{array}{c}\mathrm{Lu}(\mathrm{a}+) \\
\operatorname{Le}(\mathrm{a}-) \\
\mathrm{A}_{\mathbf{z}}\end{array}$ & $\begin{array}{c}\operatorname{Lu}(a+) \\
\operatorname{Le}(a-) \\
O\end{array}$ & $\begin{array}{r}\operatorname{Lu}(a-) \\
\operatorname{Le}(a-) \\
A_{z}\end{array}$ & $\begin{array}{c}\operatorname{Lu}(a-) \\
\operatorname{Le}(a-) \\
O\end{array}$ & $\begin{array}{c}\text { dissimilar } \\
\text { quadruplets }\end{array}$ \\
\hline 35 & $\begin{array}{c}\operatorname{Lu}(a-) \\
\operatorname{Le}(a-) \\
A_{1}\end{array}$ & $\begin{array}{c}\mathrm{Lu}(\mathrm{a}+) \\
\mathrm{Le}(\mathrm{a}+\mathrm{b}-) \\
\mathrm{O}\end{array}$ & $\begin{array}{c}\mathrm{Lu}(\mathrm{a}+) \\
\mathrm{Le}(\mathrm{a}-) \\
\mathrm{O}\end{array}$ & $\begin{array}{c}\mathrm{Lu}(\mathrm{a}-) \\
\mathrm{Le}(\mathrm{a}-) \\
\mathrm{O}\end{array}$ & & & & & \\
\hline 36 & $\begin{array}{c}\mathrm{Lu}(\mathrm{a}+) \\
\mathrm{Le}(\mathrm{a}+) \\
\mathrm{B} \\
\end{array}$ & $\begin{array}{c}\operatorname{Lu}(a-) \\
\operatorname{Le}(a-) \\
A_{1}\end{array}$ & $\begin{array}{c}\overline{\operatorname{Lu}(a+)} \\
\operatorname{Le}(\mathrm{a}-) \\
\mathrm{O}\end{array}$ & $\begin{array}{c}\mathrm{Lu}(a-) \\
\operatorname{Le}(a-) \\
B\end{array}$ & $\begin{array}{c}\mathrm{Lu}(\mathrm{a}-) \\
\mathrm{Le}(\mathrm{a}-) \\
B \\
\end{array}$ & $\begin{array}{c}\mathrm{Lu}(\mathrm{a}+) \\
\text { sec. } \\
\mathrm{Le}(\mathrm{a}-) \\
\mathrm{L} \\
\mathrm{A}_{1} \mathrm{~B} \\
\end{array}$ & & & \\
\hline 37 & $\begin{array}{l}\operatorname{Lu}(a-) \\
\operatorname{Le}(a+b-) \\
0\end{array}$ & & $\begin{array}{c}\mathrm{Lu}(\mathrm{a}+) \\
\mathrm{Le}(\mathrm{a}-\mathrm{b}-) \\
\mathrm{O}\end{array}$ & $\begin{array}{c}\operatorname{Lu}(a-) \\
\operatorname{Le}(a-b+) \\
A_{1}\end{array}$ & dissimila & ar twins & & & \\
\hline $3^{8}$ & $\begin{array}{c}\mathrm{Lu}(\mathrm{a}-) \\
\text { sec. } \\
\mathrm{Le}(\mathrm{a}-\mathrm{b}-) \\
\text { Il } \\
O\end{array}$ & & $\begin{array}{c}\operatorname{Lu}(a+) \\
\operatorname{Le}(a-b+) \\
0\end{array}$ & $\begin{array}{c}\operatorname{Lu}(a-) \\
\text { sec. } \\
\mathrm{Le}(a-b+) \\
L \\
\mathrm{~A}_{2} \\
\end{array}$ & & & & & \\
\hline 39 & $\begin{array}{c}\mathrm{Lu}(\mathrm{a}+\mathbf{)} \\
\mathrm{A}_{\mathbf{1}}\end{array}$ & $\begin{array}{c}\operatorname{Lu}(a+) \\
\operatorname{Le}(a-b+) \\
A B\end{array}$ & $\begin{array}{c}\operatorname{Lu}(a-) \\
\operatorname{Le}(a+b-) \\
A B\end{array}$ & $\begin{array}{c}\mathrm{Lu}(\mathrm{a}+) \\
\mathrm{Le}(\mathrm{a}-\mathrm{b}+) \\
\mathrm{A}_{1} \mathrm{~B}\end{array}$ & $\begin{array}{c}\operatorname{Lu}(a+) \\
\operatorname{Le}(a-b+) \\
B\end{array}$ & & & & \\
\hline 40 & $\begin{array}{l}\text { sec. } \\
\text { L }\end{array}$ & $\begin{array}{c}\operatorname{Lu}(a+) \\
\text { sec. } \\
\operatorname{Le}(a-b+) \\
L \\
O\end{array}$ & \begin{tabular}{|c|}
$\operatorname{Lu}(a-)$ \\
sec. \\
$\operatorname{Le}(a-b+)$ \\
$L$ \\
$O$
\end{tabular} & \begin{tabular}{|c|}
$\operatorname{Lu}(a-)$ \\
sec. \\
$\operatorname{Le}(a-b+)$ \\
$L$ \\
$O$
\end{tabular} & & & & & \\
\hline
\end{tabular}

Some of the unscored families could be scored but the results would be negligible. Some of the families $\left(e . g \cdot 3^{1} \cdot 3^{6}\right)$ are probably unscorable but we thought it better to include them.

- The mother in family $g$ is heterozygous $L u^{a} L_{u^{b}}$ because her mother is $L u^{b} L u^{b}$ $\mathrm{L}=\mathrm{Le}^{\mathrm{a}}$ substance present in the saliva : $11=\mathrm{Le}^{\mathrm{a}}$ substance absent. 
For more subtle analysis of the families shown in table 2 the following Caucasian gene frequencies will be needed :

$$
\begin{aligned}
& \begin{array}{lllll}
L u^{a} & 0.0390 \quad S e & 0.5077 & L & 0.6870
\end{array} \\
& L u^{b} \text { o.96 Iо se } 0.4923 \quad l \quad 0.3130
\end{aligned}
$$

The $L u^{a}$ and $L u^{b}$ frequencies are taken from Race and Sanger (1958);

TABLE 3

\begin{tabular}{|c|c|c|c|c|c|}
\hline Comparisons & $\begin{array}{l}\text { Families } \\
\text { scored }\end{array}$ & $\Sigma(\lambda)$ & $\Sigma(\kappa)$ & $2 \cdot 33 \sqrt{\Sigma(\kappa)}$ & $\frac{1}{2}\left[\mathrm{I}-\sqrt{\frac{\Sigma(\lambda)}{\Sigma(\kappa)}}\right]$ \\
\hline $\begin{array}{l}\text { Lutheran : secretor } \\
\text { Lutheran : } L l \\
\text { Secretor : } L l \\
\text { Lutheran : Le } \\
\text { type of red cells }\end{array}$ & $\begin{array}{c}\mathrm{I}, 2,3, \mathrm{I} \mathrm{I}, \mathrm{I} 3 \\
\mathrm{I} 3 \text { and } \mathrm{I} 9 \\
\mathrm{I} 3 \\
\mathrm{I}, 2,3,4,5 \\
7,8,9,10 \\
\mathrm{I}, \mathrm{I} 2\end{array}$ & $\begin{array}{r}+17.172 \\
-1.387 \\
+0.057 \\
+19.792\end{array}$ & $\begin{array}{r}34 \cdot 66 \text { o } \\
2 \cdot 232 \\
1 \cdot 059 \\
38 \cdot 667\end{array}$ & $\begin{array}{r}13.7 \\
3.5 \\
2.4 \\
14.5\end{array}$ & $\begin{array}{c}0.15 \\
\ldots \\
\ldots \\
0.14\end{array}$ \\
\hline
\end{tabular}

Linkage analysis of the "certain" families of table 2

TABLE 4

\begin{tabular}{|c|c|c|c|c|c|c|}
\hline Comparison & \multicolumn{2}{|c|}{ Source } & $\Sigma(\lambda)$ & $\Sigma(\kappa)$ & $2 \cdot 33 \sqrt{\Sigma(\kappa)}$ & $\frac{I}{0}\left[\mathrm{I}-\sqrt{\frac{\overline{\Sigma(\lambda)}}{\Gamma(\lambda)}}\right]$ \\
\hline Lutheran : secretor . & Present paper & . & $+17 \cdot 17^{2}$ & $34 \cdot 660$ & 13.7 & 0.15 \\
\hline \multirow[t]{2}{*}{ Lutheran : $L l$. } & \multirow{2}{*}{\multicolumn{2}{|c|}{$\begin{array}{c}\text { Ceppellini and Siniscalco } \\
\text { Present paper } \\
\text { Total }\end{array}$}} & $\begin{array}{l}-0.556 \\
-1.387\end{array}$ & $\begin{array}{l}4 \cdot 333 \\
2 \cdot 232\end{array}$ & \multirow[b]{2}{*}{$6 \cdot 0$} & \\
\hline & & & $-I \cdot 943$ & $6 \cdot 565$ & & \\
\hline \multirow[t]{2}{*}{ Secretor : $L l$. } & $\begin{array}{l}\text { Ceppellini and } \\
\text { Present paper }\end{array}$ & $\begin{array}{l}\text { niscalco } \\
. \quad .\end{array}$ & $\begin{array}{l}-3 \cdot 556 \\
+0 \cdot 057\end{array}$ & $\begin{array}{r}4 I \cdot 35^{8} \\
\mathrm{I} \cdot 059\end{array}$ & \multirow[b]{2}{*}{ I $5 \cdot 2$} & \\
\hline & Total & . & $-3 \cdot 499$ & $42 \cdot 4^{17}$ & & \\
\hline \multirow[t]{2}{*}{$\begin{array}{l}\text { Lutheran : Le } e^{\mathrm{q}} \text { pheno- } \\
\text { type of red cells }\end{array}$} & $\begin{array}{l}\text { Mohr } \\
\text { Present paper }\end{array}$ & $\dot{.}$ & $\begin{array}{l}+48 \cdot 965 \\
+19 \cdot 79^{2}\end{array}$ & $\begin{array}{l}62 \cdot 5 j 6 \\
38 \cdot 667\end{array}$ & \multirow[b]{2}{*}{23.4} & \multirow[b]{2}{*}{0.09} \\
\hline & Total & . & $+68 \cdot 757$ & $101 \cdot 223$ & & \\
\hline
\end{tabular}

The linkage results of Mohr and of Ceppellini and Siniscalco added to our own

the $S e$ and se frequencies are taken from Clarke et al. (1956); the $L$ and $l$ frequencies are taken from Grubb (I95I). The following approximate red cell phenotype frequencies may also be of use : $\operatorname{Le}(a+b-) 22$ per cent., $\operatorname{Le}(a-b+) 72$ per cent., $\operatorname{Le}(a-b-) 6$ per cent. 


\section{DISCUSSION}

Table 4 shows that the Lutheran and secretor genes are linked: it also shows that the Lutheran genes are linked to genes responsible for the Lewis phenotypes of the red cells. According to the present view the Lewis phenotypes of the red cells are controlled by the $L l$ genes and the secretor genes. The evidence against linkage between the $L l$ genes and the secretor genes is weighty. Therefore the linkage between the Lutheran genes and the Lewis phenotype of the red cells can most economically be explained by the Lutheran-secretor linkage: there is no need to postulate linkage between the Lutheran genes and the $L l$ genes and, indeed, the direct evidence against such linkage is slowly being collected. The collection is bound to be slow for the only really informative type of mating, $L u^{a} L u^{b}, L l \times L u^{b} L u^{b}$, $l l$ has a frequency in England of three in a thousand, and, to contribute usefully the mating would have to have produced, at the very least, four children.

At present then it seems certain that the Lutheran genes are linked to the secretor genes and most unlikely that they are linked to the Lewis genes as we now understand them.

\section{SUMMARY}

The first autosomal linkage to be recognised in Man was that discovered by Mohr between the Lutheran genes and genes responsible for the $\operatorname{Le}\left(\mathrm{a}_{+}\right)$and $\operatorname{Le}(\mathrm{a}-)$ reactions of red cells. The linkage gains support from our results which, when combined with those of Mohr, suggest a recombination fraction of 0.09 .

It is now becoming clear that the linkage is in fact between the Lutheran genes and the secretor genes and not between the Lutheran and Lewis $(L l)$ genes. The appearance of linkage with the Lewis genes is due to their phenotypic expression on red cells being controlled by the secretor genes.

Acknowledgments.-We wish to thank Dr W. G. D. Murray, of Greenhithe, and Dr C. A. Holman, of the Lewisham Hospital, for the samples of blood and saliva from family No. I. We are grateful to the family of Professor C. D. Darlington, F.R.s. (family No. 2) for giving their blood and for having such informative groups.

Past and present members of the Unit responsible for some of the family groupings are Dr Sylvia D. Lawler, Mrs Joan Sneath, Miss Helene Holt, Miss Phyllis Moores and Miss Jean Noades.

\section{REFERENCES}

CEPPELlinI, R. I955a. Nuova interpretazione sulla genetica dei caratteri Lewis eritrocitari e salivari derivante dall'analisi di 87 famiglie. Supplement to Ric. sci. Mem., 25, 3-9 (in offprint).

CEPPELLINI, R. I $955 b$. On the genetics of secretor and Lewis characters : a family study. Proc. Vth Int. Cong. Blood Transfusion, Paris, pp. 207-2II.

CEPPELLINI, R., AND SINISCALCO, M. I 955. Una nuova ipotesi genetica per il sistema Lewis-Secretore e suoi riflessi nei riguardi di alcune evidenze di linkage con altri loci. Rivista dell'Istituto Sieroterapico Italiano, 30, 43 1-445. 
ClARKe, C. A., EDWARDS, J. W., HADDOCK, D. R. W., HOWEL-EVANS, A. W., MCCONNELl, R. B., AND SHEPPARD, P. M. I956. ABO blood groups and secretor character in duodenal ulcer. Population and sibship studies. Brit. med. J., 2, 725-731. CUTBUSH, MARIE, AND CHANARIN, I. I956. The expected blood-group antibody, anti-Lub. Nature, Lond., ${ }^{\mathrm{b}} 78,855-856$.

Finney, D. J. I940. The detection of linkage. Ann. Eugen., Lond., Io, I 7 I-2 I 4 .

FISHER, R. A. I935. The detection of linkage with "dominant" abnormalities. Ann. Eugen., Lond., 6, I87-201.

GRUBB, R. 1948. Correlation between Lewis blood group and secretor character in man. Nature, Lond., $162,933$.

GRUBB, R. I950. Quelques aspects de la complexité des groupes ABO. Rev. Hémat., 5, 268-275.

GRUBB, R. I95I. Observations on the human group system Lewis. Acta path. microbiol. scand., 28, 6I $-8 \mathrm{I}$.

MÄKEL ̈̈, O., AND MÄKELÄ, PIRJo. I956. Le ${ }^{b}$ antigen. Studies on its occurrence in red cells, plasma and saliva. Ann. Med. exp. Fenn., 34, 157-162.

MOHR, J. I 95 I $a$. A search for linkage between the Lutheran blood group and other hereditary characters. Acta path. microbiol. scand., 28, 207-2 I0.

MOHR, J. I 95 I $b$. Estimation of linkage between the Lutheran and the Lewis blood groups. Acta path. microbiol. scand., 29, 339-344.

мoHr, J. 1954. A Study of Linkage in Man. Munksgaard, Copenhagen.

MoHR, J. 1956. To what extent has linkage between various human blood group systems been excluded? Acta genet., 6, 24-34.

RACE, R. R., AND SANGer, RUTH. I954. Blood Groups in Man. 2nd ed. Blackwell Scientific Publications, Oxford.

RACE, R. R., AND SANGer, RUTH. 1958. Blood Groups in Man. 3rd ed. Blackwell Scientific Publications, Oxford.

SNEATH, JOAN S., AND SNEATH, P. H. A. I955. Transformation of the Lewis groups of human red cells. Nature, Lond., 176, i 72. 